\title{
Assessing the Reliability and Validity of a Newly Developed Breathing Movement Measuring Device
}

\author{
Hideo KANEKo, PT, $\mathrm{PhD}^{1)}$ \\ 1) Department of Physical Therapy, Fukuoka School of Rehabilitation Sciences, International \\ University of Health and Welfare: 137-1 Enokizu, Okawa-shi, Fukuoka 831-8501, Japan. \\ TEL +81 944-89-2000
}

\begin{abstract}
Purpose] The purpose of this study was to test the reliability and validity of a breathing movement measuring device (BMMD) that was newly developed for the objective assessment of breathing movements in clinical practice. [Subjects and Methods] The breathing movement distances of the chest (bilateral third and eighth ribs) and abdomen (upper abdomen) of 30 healthy young males were measured using the BMMD and a threedimensional (3D) motion analysis system during quiet breathing. During measurements, the pen-size mechanical BMMD was held in the hand and placed on selected observation points. Distances on the BMMD scale were read by two raters. In addition, the breathing movement distances of 10 subjects were measured during quiet breathing using the BMMD fixed on a tripod. The distances between the reflective markers on the observation points were measured. The intraclass correlation coefficient, Bland-Altman analysis, and Pearson's correlation coefficient were performed. [Results] The inter-rater reliability of the BMMD was low-to-moderate. However, the reliability and validity between the two measurement methods were high. Systematic errors were identified for almost all the observation points. [Conclusion] The reliability and validity of the BMMD, which works by comparing the reflective marker distances, were acceptable. These findings suggest that the BMMD may be useful for quantitatively assessing chest and abdominal wall movements during quiet breathing in clinical practice.

Key words: Reliability, Validity, Breathing movement measuring device
\end{abstract}

(This article was submitted Oct. 29, 2012, and was accepted Nov. 28, 2012)

\section{INTRODUCTION}

Breathing movement is one of the important assessments of pulmonary function. In clinical practice, breathing movement is generally evaluated during physical examination by inspection and palpation, which provide real-time observations and do not require a special measuring instrument. However, physical examinations are not quantitative and depend on the experience of the assessor. Although precision measurement equipment such as a magnetometers ${ }^{1)}$, respiratory inductive plethysmography ${ }^{2}$, optoelectronic plethysmography ${ }^{3-7)}$, and respiratory movement measuring instrument ${ }^{8)}$ can be used for quantitative measurements, these devices are unsuitable for clinical practice because of their high cost and complexity. Therefore, a breathing movement scale that assesses five levels of breathing movements of the chest and abdominal wall by physical examination was developed. On this scale, breathing movements are divided into three sections (upper chest, lower chest, and abdomen), and the criterion for breathing movement distances at the assessment point of each section has been specified on the basis of normal breathing movement ${ }^{9}$. However, in our preliminary study of the breathing movement scale, we found that its reliability was not sufficient because the assessment required physical examinations by inspection and palpation

E-mail: hkaneko@iuhw.ac.jp which depend on the experience of the assessor. Therefore, for more objective assessment of breathing movements in clinical practice, the breathing movement measuring device (BMMD) was developed. This study was conducted to test the reliability and validity of the BMMD.

\section{SUBJECTS AND METHODS}

Thirty healthy young males with an average age of 22 \pm 1 (standard deviation, SD) years; average height of 1.69 $\pm 0.05 \mathrm{~m}$; and average weight of $61 \pm 7 \mathrm{~kg}$ participated in this study. The subjects were excluded if they had a history of respiratory, circulatory, or neurological disorders. The study was approved by the local ethics committee, and all subjects provided their informed consent.

The BMMD (length, $12 \mathrm{~cm}$ ) comprises a syringe with a millimeter scale, angle gauge, a tension spring, a metal rod, a coupling part with a mark, and a discoid contact part with a ball joint. The tension spring is fixed in the syringe, and the tension spring and metal rod are connected using the coupling part. The discoid contact part is joined to the other end of the metal rod. The breathing movement distance from observation points can be estimated from the position of the mark which moves on the scale through the discoid contact (Fig. 1).

Using a 3D motion analysis system (Vicon MX, Vicon Motion System Ltd., Oxford, UK), breathing movements were measured during quiet breathing while subjects were 
in the supine position. This system comprises eight infrared cameras that track the movement trajectories of 14-mm passive markers attached to the chest and abdominal wall. Camera images are sampled at a rate of $50 \mathrm{~Hz}$, and the motion data are processed using software (Vicon Workstation 5.2.4, Vicon Motion System Ltd.) to produce 3D coordinates for each marker. These coordinates were smoothed using a Woltring filter, and the distances between the markers were calculated using a spreadsheet software (Excel, Microsoft, Redmond, WA, USA). Five passive markers were bilaterally placed along the vertical line through the medial onethird of the clavicle (the third rib and the eighth rib) and along the vertical line through the umbilicus (the midpoint between the xiphoid process and umbilicus, the abdomen). These points were chosen as representative parts which show greater movement of the upper chest, lower chest, and abdomen, and reflect the characteristics of normal breathing movement ${ }^{9}$. The range of movement was measured as the $3 \mathrm{D}$ distance at half the respiratory cycle. Distances were calculated and expressed in millimeters on the basis of a single breathing cycle that was measured by the two raters using the BMMD.

The subjects were advised to remain relaxed, with their shirt off and pants loosened, on a bed in the supine position. They were instructed not to talk or move during the measurements and to breathe normally to emulate a quiet breathing condition. The breathing movements during quiet breathing were measured and recorded in millimeters by two raters using the BMMD for approximately $5 \mathrm{~min}$. When measuring breathing movement distances, the BMMD was held $30^{\circ}$ cranially and slightly laterally in an inclined position to the vertical line of the chest and along the vertical line of the abdomen. This position was determined on the basis of the average measurements of the angle between the line from the start to the end of inspiration and a vertical

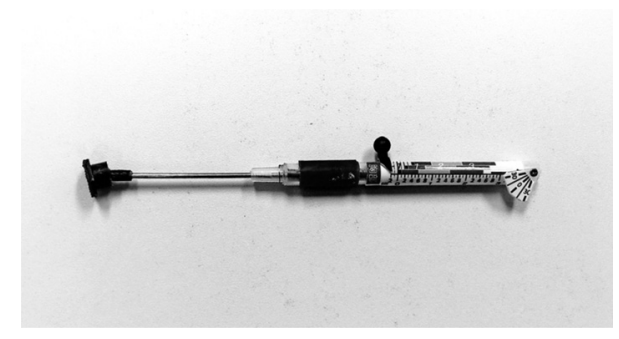

Fig. 1. Breathing movement measuring device (BMMD) line, as shown in our previous study ${ }^{9)}$.

The raters were instructed to use the lateral point of the reflective markers and stabilize the elbow by holding the BMMD on the bed. The BMMD mark was adjusted to the starting point of the scale at the end of expiration by lightly pressing the BMMD against the observation point. Breathing movements were calculated from the distance that the mark moved from the end of expiration to the end of inspiration.

Furthermore, to identify the influence of fixation on the BMMD, the distances of breathing movements of 10 subjects were measured by fixing the BMMD on a tripod with a bar that was operated by the same rater. The rater was instructed to sign immediately after each measurement to ensure that the data was synchronized with that of the $3 \mathrm{D}$ motion analysis system.

Values are expressed as means $\pm \mathrm{SD}$. The intraclass correlation coefficient (ICC) $(2,1)$ was used to identify the inter-rater reliability of measurements using the BMMD. ICC $(1,1)$ was used to calculate the test-retest reliability of measurements using the 3D motion analysis system when two raters measured breathing movement with the BMMD. Furthermore, to investigate the reliability and the criterionrelated validity between the two methods, $\operatorname{ICC}(3,1)$ and Pearson's correlation coefficient were used, respectively. Bland-Altman analysis was also performed to assess the agreement between the two methods. The minimal detectable change, mean difference, mean percentage difference between measurements, and limits of agreement were calculated. Statistical analyses were performed using statistical software (IBM SPSS Statistics 19, IBM, Armonk, NY, USA). Values of $p<0.05$ were considered statistically significant.

\section{RESULTS}

ICC $(2,1)$ for measurements using the BMMD were lowto-moderate for almost all the observation points (ICC [2, $1]=0.34-0.69)$ (Table 1). ICCs for the right third rib and the abdomen were relatively high and that for the left eighth rib was the lowest. The Bland-Altman analysis results revealed fixed errors for all the observation points except the right third rib. The mean distance measured by one rater (rater 1) was lower than that calculated by the other rater (rater 2).

For measurements of reflective marker distances when the two raters used the BMMD, ICC $(1,1)$ was $0.71-0.76$, without any systematic errors (Table 2).

For the two measurement methods, $\operatorname{ICC}(3,1)$ was high

Table 1. Inter-rater reliability of measurements using the BMMD

\begin{tabular}{llcccc}
\hline & & ICC $(2,1)$ & MDC $(\mathrm{mm})$ & Bias $\uparrow \pm$ LOA $(\mathrm{mm})$ & \%Bias \pm LOA (\%) \\
\hline \multirow{2}{*}{ Third Rib } & right & 0.69 & 1.4 & $-0.1 \pm 1.0$ & $-5 \pm 49$ \\
& left & 0.50 & 1.5 & $-0.6 \pm 1.0$ & $-31 \pm 49$ \\
\multirow{2}{*}{ Eighth Rib } & right & 0.41 & 1.4 & $-0.6 \pm 1.0^{*}$ & $-26 \pm 42$ \\
& left & 0.34 & 1.4 & $-0.6 \pm 1.0^{*}$ & $-24 \pm 33$ \\
Abdomen & & 0.63 & 3.1 & $-1.1 \pm 2.1^{*}$ & $-14 \pm 32$ \\
\hline
\end{tabular}

ICC: intraclass correlation coefficient; MDC: minimal detectable change; LOA: limits of agreement; $* \mathrm{p}<0.05$; $\dagger$ Rater 1 minus Rater 2. 
for almost all the observation points for both raters $(0.65$ 0.89) (Table 3). Both raters showed fixed errors, in which the distances were lower as measured by the BMMD than those of the 3D analysis system. Proportional errors were also observed. When the BMMD was fixed on a tripod, ICC $(3,1)$ was higher $(0.77-0.93)$, and fixed errors with low values from the BMMD were identified for almost all the ob- servation points, but proportional errors were not observed. The correlation coefficients between the BMMD and the reflective markers were $0.72-0.93$ for rater $1,0.66-0.92$ for rater 2 , and $0.79-0.97$ for the fixed condition. The criterionrelated validity for almost all the observation points was high $(r=0.68-0.94)$ (Table 4).

Table 2. Test-retest reliability of measurements using the 3D motion analysis system

\begin{tabular}{llcccc}
\hline & & ICC $(1,1)$ & MDC $(\mathrm{mm})$ & Bias \pm LOA $(\mathrm{mm})$ & \%Bias \pm LOA (\%) \\
\hline \multirow{2}{*}{ Third Rib } & right & 0.77 & 1.6 & $-0.1 \pm 1.1$ & $-18 \pm 20$ \\
& left & 0.72 & 1.7 & $-0.2 \pm 1.1$ & $-27 \pm 27$ \\
Eighth Rib & right & 0.71 & 1.6 & $-0.2 \pm 1.0$ & $-25 \pm 19$ \\
& left & 0.71 & 1.6 & $-0.0 \pm 1.1$ & $-30 \pm 19$ \\
Abdomen & & 0.76 & 3.6 & $0.3 \pm 2.4$ & $-29 \pm 12$ \\
\hline
\end{tabular}

ICC: intraclass correlation coefficient; MDC: minimal detectable change; LOA: limits of agreement.

Table 3. Reliability of measurements between the distances of the BMMD and the $3 \mathrm{D}$ motion analysis system for each rater $(n=30)$ and the fixation condition $(\mathrm{n}=10)$

\begin{tabular}{|c|c|c|c|c|c|c|c|}
\hline & & BMMD (mm) & 3D (mm) & $\operatorname{ICC}(3,1)$ & $\mathrm{MDC}(\mathrm{mm})$ & Bais \pm LOA (mm) & $\% \operatorname{Bias} \pm$ LOA $(\%)$ \\
\hline \multicolumn{8}{|l|}{ Rater 1} \\
\hline \multirow{2}{*}{ Third Rib } & right & $1.8 \pm 0.9$ & $2.2 \pm 1.1$ & 0.88 & 1.0 & $-0.3 \pm 0.7^{*}$ & $-10 \pm 33^{*}$ \\
\hline & left & $1.6 \pm 0.8$ & $2.4 \pm 1.2$ & 0.75 & 1.5 & $-0.7 \pm 0.8^{*}$ & $-32 \pm 42 *$ \\
\hline \multirow{2}{*}{ Eighth Rib } & right & $2.3 \pm 0.8$ & $3.1 \pm 1.1$ & 0.71 & 1.5 & $-0.6 \pm 1.0^{*}$ & $-22 \pm 38^{*}$ \\
\hline & left & $2.3 \pm 0.7$ & $3.0 \pm 1.1$ & 0.72 & 1.4 & $-0.7 \pm 0.9^{*}$ & $-23 \pm 35^{*}$ \\
\hline Abdomen & & $7.1 \pm 2.0$ & $9.0 \pm 2.5$ & 0.88 & 2.2 & $-1.9 \pm 1.5^{*}$ & $-23 \pm 18^{*}$ \\
\hline \multicolumn{8}{|l|}{ Rater 2} \\
\hline \multirow{2}{*}{ Third Rib } & right & $1.9 \pm 1.0$ & $2.3 \pm 1.3$ & 0.78 & 1.6 & $-0.2 \pm 1.0$ & $-3 \pm 66^{*}$ \\
\hline & left & $2.3 \pm 0.9$ & $2.2 \pm 1.1$ & 0.81 & 1.2 & $-0.1 \pm 1.0$ & $-10 \pm 37^{*}$ \\
\hline \multirow{2}{*}{ Eighth Rib } & right & $3.0 \pm 0.7$ & $3.3 \pm 1.0$ & 0.78 & 1.0 & $-0.3 \pm 0.7^{*}$ & $-7 \pm 21^{*}$ \\
\hline & left & $2.9 \pm 0.7$ & $3.1 \pm 0.9$ & 0.65 & 1.2 & $-0.1 \pm 0.8$ & $-1 \pm 28^{*}$ \\
\hline Abdomen & & $8.1 \pm 2.1$ & $8.6 \pm 2.7$ & 0.89 & 2.2 & $-0.5 \pm 1.5^{*}$ & $-5 \pm 19^{*}$ \\
\hline \multicolumn{8}{|l|}{ Fixation } \\
\hline \multirow{2}{*}{ Third Rib } & right & $1.8 \pm 1.1$ & $2.2 \pm 1.4$ & 0.92 & 1.0 & $-0.4 \pm 0.4^{*}$ & $-18 \pm 20$ \\
\hline & left & $1.9 \pm 1.2$ & $2.3 \pm 0.8$ & 0.77 & 1.4 & $-0.4 \pm 0.5$ & $-28 \pm 27$ \\
\hline \multirow{2}{*}{ Eighth Rib } & right & $2.4 \pm 1.2$ & $3.0 \pm 1.2$ & 0.91 & 1.0 & $-0.6 \pm 0.4^{*}$ & $-25 \pm 19$ \\
\hline & left & $2.2 \pm 0.9$ & $3.1 \pm 1.4$ & 0.80 & 1.4 & $-0.9 \pm 0.5^{*}$ & $-31 \pm 19$ \\
\hline Abdomen & & $7.2 \pm 2.7$ & $9.4 \pm 2.8$ & 0.93 & 2.0 & $-2.2 \pm 0.7^{*}$ & $-23 \pm 18$ \\
\hline
\end{tabular}

BMMD: breathing movement measuring device; ICC: intraclass correlation coefficient; MDC: minimal detectable change; LOA limits of agreement. * $\mathrm{p}<0.05$

Table 4. Criterion-related validity between the distances of the BMMD and the 3D motion analysis system for each rater $(n=30)$ and fixation condition $(\mathrm{n}=10)$

\begin{tabular}{llccc}
\hline & & Fixation & Rater 1 & Rater 2 \\
\hline \multirow{2}{*}{ Third Rib } & right & $0.94^{*}$ & $0.91^{*}$ & $0.84^{*}$ \\
& left & $0.82^{*}$ & $0.81^{*}$ & $0.84^{*}$ \\
\multirow{2}{*}{ Eighth Rib } & right & $0.91^{*}$ & $0.74^{*}$ & $0.84^{*}$ \\
& left & $0.87^{*}$ & $0.80^{*}$ & $0.68^{*}$ \\
Abdomen & & $0.93^{*}$ & $0.90^{*}$ & $0.93^{*}$ \\
\hline
\end{tabular}

BMMD: breathing movement measuring device; Fixation: the BMMD fixed with a tripod; $* \mathrm{p}<0.05$ 


\section{DISCUSSION}

This study investigated the reliability and validity of the newly developed BMMD. One motive for developing the BMMD was the high degree of subjectivity existing in breathing movement assessments in current clinical practice. Although the inter-rater reliability of the distance measured using the BMMD was not optimal, the reliability and validity of the BMMD, compared to the results of the $3 \mathrm{D}$ motion analysis system, were high. These findings suggest that the simple BMMD provides a feasible method for the objective assessment of breathing movements of the chest and abdominal wall in clinical practice.

Our previous study showed that the intra- and inter-rater reliabilities of the marker distance measurements were acceptable and the 3D distances of the chest and abdominal markers do represent the variable features of breathing movements among the healthy subjects 20-74 years of age $^{9)}$. We assume that the data would be useful for observational assessments of breathing movement during physical examinations. Therefore, the breathing movement scale that assesses five levels of breathing movements of the chest and abdominal wall by physical examination was developed. However, in a preliminary study, we found that the reliability of the breathing movement scale currently used in physical examinations was insufficient. Therefore, The BMMD was developed on the basis of the data collected from normal breathing movement distances ${ }^{9}$, in an effort to increase the reliability of the breathing movement scale. In this study, the BMMD reliability and validity were high, particularly when the device was fixed. In addition, fixed errors had lower distances with the BMMD for almost all the observation points. These results were assumed to be because of the following reasons: 1) Trajectories of the observation points on the chest and abdominal wall are not linear, whereas the distances measured using the BMMD are linear. 2) Soft tissues at observational points may be dented by compression and shear force generated by the tension of the spring in accordance with respiratory movement. In fact, the fixed error was highest at the abdomen rather than the chest. 3) Strictly speaking, the observation points of the BMMD and the reflective markers are not identical, i.e., the BMMD was located slightly more laterally than the reflective markers. 4) There are differences in breathing movement directions between the subjects and the BMMD. In fact, a wide variation has been reported regarding breathing patterns in normal supine subjects ${ }^{10)}$. Therefore, as the difference in breathing movement angle becomes larger, shear force may increase resulting in a decrease in the distance of the BMMD.

As noted above, fixed errors were observed in rater measurements and the fixation device. However, the ranges of limits of agreement (LOA) with the fixation device were approximately the smallest unit during quiet breathing ( $<1.0 \mathrm{~mm}$ for the chest and $1.5 \mathrm{~mm}$ for abdomen), and the LOA ranges without the fixation device were lower than the minimal distance between the breathing movement scales ( $2.5 \mathrm{~mm}$ for the chest and $4.0 \mathrm{~mm}$ for abdomen) which were determined on the basis of the data from normal breathing movement ${ }^{9}$. Given that $\operatorname{ICC}(3,1)$ and correlation coefficients were high, these results are acceptable for clinical practice.

On the other hand, the inter-rater reliability of the BMMD was low-to-moderate. One possible explanation for this is that quiet breathing movements are variable. Actually, in this study, ICC $(1,1)$ of reflective marker distances when the two raters used the BMMD were $0.71-0.77$, and that the minimal detectable change exceeded the ranges of LOAs of each observation point. In addition, ICC $(1,1)$ in this study was lower than that in our previous study ${ }^{9}$, in which the distances were calculated on the basis of the average values of five steady breathing cycles during quiet breathing. The lower ICC $(1,1)$ may be attributable to the number of analyzed breathing movements. In this study, single breathing cycles were analyzed. Therefore, when measuring breathing movements using the BMMD, breathing movement distances should be estimated by observing some steady breathing cycles. Further studies need to be conducted to verify the inter-rater reliability of the BMMD.

In clinical practice, an objective measurement of breathing movement may promote a more defined evaluation of respiratory status which can be shared among healthcare professionals. It is considered that the BMMD may be helpful for the objective assessment of breathing movements in clinical practice, because we found that the results produced by this device have a high degree of reliability and validity. In addition, the BMMD is portable and does not need a continuous flow of electricity. If the device is not fixed, it is necessary to rest the elbow to stabilize the BMMD. Moreover, breathing movements should be estimated by observing several breathing cycles because the reliability of measurements from a single breathing cycle is insufficient.

These results did not include data from subjects with increased respiratory movement, as found in patients with respiratory dysfunctions. Although the BMMD may be useful as a device assisting assessment with the breathing movement scale to enhance objective measurements, further studies are needed to identify the BMMD reliability for subjects with increased respiratory movement, and to consider its limitations.

\section{ACKNOWLEDGEMENT}

This work was supported by the Japan Society for the Promotion of Science Grant-in-Aid for Scientific Research, 22500472.

\section{REFERENCES}

1) Sharp JT, Goldberg NB, Druz WS, et al.: Relative contributions of rib cage and abdomen to breathing in normal subjects. J Appl Physiol, 1975, 39: 608-618. [Medline]

2) Gilbert R, Auchincloss JH Jr, Peppi D: Relationship of rib cage and abdomen motion to diaphragm function during quiet breathing. Chest, 1981, 80: 607-612. [Medline] [CrossRef]

3) Cala SJ, Kenyon CM, Ferrigno G, et al.: Chest wall and lung volume estimation by optical reflectance motion analysis. J Appl Physiol, 1996, 81: 2680-2689. [Medline]

4) Aliverti A, Dellaca R, Pelosi P, et al.: Optoelectronic plethysmography in intensive care patients. Am J Respir Crit Care Med, 2000, 161: 1546-1552. 
[Medline]

5) Aliverti A, Dellaca R, Pelosi P, et al.: Compartmental analysis of breathing in the supine and prone positions by optoelectronic plethysmography. Ann Biomed Eng, 2001, 29: 60-70. [Medline] [CrossRef]

6) Binazzi B, Lanini B, Bianchi R, et al.: Breathing pattern and kinematics in normal subjects during speech, singing and loud whispering. Acta Physiol (Oxf), 2006, 186: 233-246. [Medline] [CrossRef]

7) Romei M, Mauro AL, D'Angelo MG, et al.: Effects of gender and posture on thoraco-abdominal kinematics during quiet breathing in healthy adults.
Respir Physiol Neurobiol, 2010, 172: 184-191. [Medline] [CrossRef]

8) Ragnarsdóttir M, Kristinsdottir EK: Breathing movements and breathing patterns among healthy men and women 20-69 years of age. Reference values. Respiration, 2006, 73: 48-54. [Medline] [CrossRef]

9) Kaneko H, Horie J: Breathing movements of the chest and abdominal wall in healthy subjects. Respir Care, 2012, 57: 1442-1451. [Medline]

10) Tobin MJ, Chadha TS, Jenouri G, et al.: Breathing patterns. 1. Normal subjects. Chest, 1983, 84: 202-205. [Medline] [CrossRef] 